\title{
LA APLICACIÓN DE LA EUTANASIA EN ESPAÑA: UN ESTUDIO SOBRE LA PROPOSICIÓN DE LA LEY ORGÁNICA DE LA EUTANASIA DEL PARTIDO SOCIALISTA OBRERO ESPAÑOL
}

\author{
THE APPLICATION OF EUTHANASIA IN SPAIN: A STUDY ON THE \\ PROPOSITION OF THE ORGANIC LAW OF THE EUTHANASIA OF THE \\ SOCIALIST PARTY SPANISH WORKER
}

Salvador Morales Ferrer*

\begin{abstract}
Resumen: Desde principios del siglo XXI, siempre en los Hospitales Españoles sea practicado los cuidados paliativos a las personas enfermas en fase terminal, para que estas en su último momento de su vida no sufran, a todo esto, cabe mencionar que no existe una regulación legal muy específica sobre esta materia en España, sin embargo, cabe la posibilidad tanto del enfermo terminal o, sus familiares incluso el médico que pueda permitir su aplicación. Lo que intenta el legislador Socialista en la Proposición de Ley Orgánica de la Eutanasia consiste en la práctica directa de quién la practica ya sea un profesional de la medicina o incluso un familiar al enfermo terminal sin carácter punitivo mediante el consentimiento tanto del paciente o, sus familiares que están a cargo de la persona enferma que ésta en fase terminal. Por lo que, se entiende que es un gran avance legislativo para España e incluso para los países del entorno.
\end{abstract}

Palabras clave: Eutanasia; España; Ley Orgánica de la eutanasia.

Resumo: Desde o início do século XXI, os hospitais espanhóis praticam os cuidados paliativos às pessoas doentes em uma fase terminal, para que estas no último momento de sua vida não sofram, todo o caso, é importante registrar que não existe regulamentação legal muito específica sobre essa matéria na Espanha. Assim, resta a possibilidade de autorizar possibilidade ao enfermo terminal, seus familiares, ou o médico. O que pretende o legislador Socialista na proposta de Lei Orgânica da Eutanásia consiste na possibilidade de que essa prática realizada seja por profissional médico ou por familiar do enfermo, desde que mediante o consentimento do doente ou de seus familiares sem que seja considerada crime. Compreende-se que essa proposta é um grande avanço legislativo para a Espanha e para os seus países vizinhos.

Palavras-chave: Eutanásia; Espanha; Lei Orgânica da Eutanásia.

\footnotetext{
* Doctor en Derecho por el programa de Estudios Jurídicos, Ciencia Política y Criminología de la Universidad de Valencia, con la calificación Apto Cum Laude; título de Experto en Mediación Familiar, Penal y Penitenciario, por la Universidad Cardenal Herrera CEU; licenciado en Derecho especialidad en Derecho Privado por la Universidad Cardenal Herrera CEU de Valencia; certificado-diploma de Estudios Avanzados Tercer Ciclo - Doctorado por la Universidad Cardenal Herrera CEU de Valencia; certificado de Aptitud Profesional realizado en la Escuela de Práctica Jurídica del Ilustre Colegio de abogados de Alzira; certificado de Aptitud Pedagógica por la Universidad de Valencia; abogado Colegiado en el Ilustre Colegio de Abogados de Alzira; letrado especialista para actuar en la Jurisdicción de Menores.
} 
La aplicación de la eutanasia en España: un estudio sobre la proposición de la Ley Ogánica de la Eutanasia del Partido Socialista Obrero Español

Abstract: Since the beginning of the 21st century, always in the Spanish Hospitals palliative care is practiced to the sick people in the terminal phase, so that at the last moment of their life they do not suffer, to all this, it is possible to mention that there is not a very legal regulation specific on this matter in Spain, however, it is possible for the terminally ill or their relatives even the doctor who can allow its application. What the Socialist legislator intends in the Organic Law Proposal of Euthanasia consists in the direct practice of those who practice it either a medical professional or even a relative to the terminal patient without punitive character through the consent of either the patient or their relatives who are in charge of the sick person who is in the terminal phase. Therefore, it is understood that it is a great legislative advance for Spain and even for the surrounding countries.

Key words: Euthanasia; Spain; Organic Law of Euthanasia.

\section{INTRODUCCIÓN}

Desde los inicios de la humanidad, siempre sea dejado sufrir a las personas enfermas en fase terminal, ya por motivos religiosos o por la cultura educacional, como menciona el autor Pereira (2016): "Como nada se sabe sobre a morte, nada se pode dizer acerca do que sea uma boa morte, uma morte boa o uma má morte" (PINHEIRO PEREIRA, 2016). Así, se puede mencionar que en los inicios del Siglo XX sea intentado ayudar por medio del Estado Español a las familias que tienen un enfermo terminal mediante medicación o utensilios para atender a sus necesidades del paciente hasta llegar a su fallecimiento mediante sufrimiento del paciente.

Tras la llegada del Siglo XXI en los hospitales españoles se aplicaron los cuidados paliativos a estas personas en fase terminal bajo el consentimiento tanto del enfermo terminal, como sus familiares o incluso el médico siempre atendiendo a la Lex Artis. Ante esto algunas personas intentaron acabar con su propia vida mediante la cooperación necesaria de un familiar o amigo, considerándose un delito de suicidio que está en la actualidad en vigor mediante el artículo 143.3 del Código Penal Español que señala: "Será castigado con la pena de prisión de seis a diez años si la cooperación llegara hasta el punto de ejecutar la muerte" (ESPAÑA, 2006).

Así, mediante la Proposición de Ley Orgánica de la Eutanasia, si es aprobada por todo el arco parlamentario español, será efectiva la eutanasia en España, y está figura jurídica dejará de ser punitiva en España. Con el presente artículo se pretende realizar un análisis descriptivo en los efectos jurídicos y políticos sobre la aplicación de la Eutanasia en España. 
El artículo tiene la siguiente estructura: el primero esboza como formula la Proposición de Ley Orgánica del Partido Socialista Obrero Español la Eutanasia en la sociedad española; el segundo presenta como se aplicaría la proposición de Ley de la Eutanasia; el tercero muestra los distintos tipos de eutanasia; el cuarto comprende el consentimiento informado tanto para el paciente que está en fase terminal así como sus familiares; el quinto se analizan los puntos del partido socialista para la aplicación de la eutanasia; sexto se muestra la aplicación en la actualidad de los cuidados paliativos y, séptimo se esboza los elementos jurídico en la Constitución Española de 1978 para la aplicación de la Eutanasia.

\section{FORMULACIÓN DE LA PROPOSICIÓN DE LEY ORGÁNICA DE LA EUTANASIA EN ESPAÑA POR EL PARTIDO SOCIALISTA OBRERO ESPAÑNL}

Hace tiempo que se asiste aún amplio debate social y político sobre los derechos y garantías de todas las personas a una asistencia sanitaria y social en el final de sus vidas, que respete su autonomía y el derecho a una muerte sin sufrimiento (muerte digna). A esto se une otro sobre el derecho de las personas que, sin estar en un proceso abocado a una muerte inminente, deciden por su situación de invalidez o sufrimiento solicitar la ayuda para morir anticipadamente (eutanasia). Dos aspectos que para el Partido Socialista Obrero Español requieren decisiones políticas que permitan avanzar en los derechos de las personas. Pero son dos debates sociales, profesionales y jurídicos bien diferentes al mismo tiempo.

En relación con la eutanasia, su regulación está llamada a solucionar los supuestos en que una persona con una enfermedad sin tratamiento, por tanto, irreversible, que sufre padecimientos graves, y que desea y solicita que se le administre una medicación que ponga final a su vida de manera anticipada o, al menos, el acceso a los fármacos para poner fin a su vida por sí mismo.

El Partido Socialista Obrero Español considera desde hace tiempo que hay un espacio (voluntad social, política y respaldo constitucional y jurídico) para legislar también en España sobre este derecho. Porque de eso se trata de una ampliación de derechos de la ciudadanía. Así quedó reflejado en el programa electoral y de manera nítida en la resolución de nuestro $39^{\circ}$ Congreso Federal. Comisión Ejecutiva Federal. De hecho, desde 2009 encuestas estadísticas se aprecia en la sociedad española un importante apoyo a la regulación legal de la eutanasia (58,4\% sí, con toda seguridad), incluso entre 
La aplicación de la eutanasia en España: un estudio sobre la proposición de la Ley Ogánica de la Eutanasia del Partido Socialista Obrero Español

los católicos (60\%) y demás creyentes $(43,7 \%)$. Con esta Proposición de Ley responde de forma serena, debatida y seria a esta demanda social.

La cuestión fundamental en esta regulación es dotarla de las garantías suficientes. La regulación de la eutanasia ha de asegurar que la decisión de la persona que solicita la eutanasia sea autónoma, libre de presiones, informada y sostenida en el tiempo, y no debida a circunstancias de la persona que tuvieran más que ver con una falta de condiciones dignas, médicas, sociales o familiares, para afrontar esa situación. Se deben valorar, además de la capacidad, autonomía y libertad de la persona, las condiciones de la enfermedad (irreversibilidad, pronóstico temporal de la muerte, sufrimientos que padece y posibilidad de alivio, ...). En consecuencia, esta no es una cuestión -ni la ley llamada a regularlo- estrictamente sanitaria, y, en consecuencia, la tutela sobre la autonomía y garantías de la decisión no han de ser tampoco de carácter únicamente sanitario. Por lo que, el legislador socialista intenta aplicar la eutanasia mediante sondeos sociales en España, para las personas que están abocadas a una muerte inminente, bajo la decisión del enfermo terminal o familiares, despenalizándola del Código Penal Español.

\section{LA APLICACIÓN DE LA PROPOSICIÓN DE LA LEY ORGÁNICA DE LA EUTANASIA DEL PARTIDO SOCIALISTA OBRERO ESPAÑOL}

Ante una enfermedad grave e incurable, definida como la que por su naturaleza origina sufrimientos físicos o psíquicos, constantes e insoportables, sin posibilidad de alivio que el paciente considere tolerable, con un pronóstico de vida limitado, en un contexto de fragilidad progresiva, lo que implica que el enfermo se encuentra en fase terminal de su vida y atendiendo a su voluntad o de la familia. Por el contrario, parte de la doctrina es contraria en este sentido cabe mencionar al autor León que menciona:

\footnotetext{
Me parece necesario, para alejar el riesgo de la confusión semántica, que todos nos olvidáramos de la noble ascendencia etimológica y de las significaciones nobles de eutanasia -buena muerte- y que, a partir de ahora, por eutanasia entendamos lisa y llanamente el matar sin dolor y deliberadamente, de ordinario mediante procedimientos de apariencia médica, a personas que se tienen como destinadas a una vida atormentada por el dolor o limitada por la incapacidad, con el propósito de ahorrarles sufrimientos o de librar a la sociedad de una carga inútil (LEON CORREA, 1997).
} 
En una discapacidad grave crónica, definida como la situación que produce en el afectado una invalidez de manera generalizada de valerse por sí mismo, sin que existan posibilidades fundadas de curación y, en cambio, sí existe seguridad o gran probabilidad de que tal incapacidad vaya a persistir durante el resto de la existencia de esa persona.

Se entienden por limitaciones aquellas que inciden fundamentalmente sobre su autonomía física y actividades de la vida diaria, así como sobre su capacidad de expresión y relación, originando por su naturaleza sufrimientos físicos o psíquicos constantes e intolerables, sin posibilidad de alivio que el paciente considere tolerable. En ocasiones puede suponer la dependencia absoluta de apoyo tecnológico. La proposición de Ley sobre la eutanasia esta mal enfocada por el legislador del Partido Socialista Español puesto que confunde discapacidad que en cierto modo sería el grado para la obtención de la dependencia.

Así, la discapacidad consiste en la atención como el elemento primordial de la misma de sus necesidades básicas de la vida diaria, como menciona el autor Rodríguez: [...] aquel estado en que se encuentran las personas que, por razones ligadas a la falta o la pérdida de autonomía física, psíquica o intelectual, tienen necesidad de asistencia y/o ayudas importantes a fin de realizar los actos corrientes de la vida diaria, actividades relativas al cuidado personal, movilidad en el hogar; tareas domésticas; recoger objetos y personas así como ejecutar órdenes sencillas, si bien no existe un completo acuerdo en la extensión del contenido de las mismas" (RODRIGUEZ CARRERO, 2008), por lo que se entiende que la persona dependiente tiene un déficit para sus actos tanto de movilidad o necesidad instrumental, necesarios para su vida y realizar sus actos vitales en su vida cotidiana.

Al respecto, la Jurisprudencia la Sentencia del Tribunal de Navarra 11 de enero de 2013 en sus II Fundamentos Jurídicos (Ponente: María Jesús Azcona Labiano) JUR 2014\44311 Aranzadi señala: “el estado de carácter permanente en que se encuentran las personas que por razones derivadas de la edad, la enfermedad o la discapacidad, y ligadas a la falta o a la pérdida de autonomía física, mental, intelectual o sensorial precisan de la atención de otra u otras personas o ayudas importantes para realizar actividades básicas de la vida diaria, o en el caso de las personas con discapacidad intelectual o enfermedad mental, de otros apoyos para su autonomía persona", al hilo la doctrina entre ellos cabe mencionar al autor Eamon O’Shea (2003) manifiesta: “es un estado en que las personas, debido a la falta o la pérdida de autonomía física, psicológica o mental, R. Fac. Dir. UFG, v. 42, n. 2, p.156-171, maio/ago. 2018 
La aplicación de la eutanasia en España: un estudio sobre la proposición de la Ley Ogánica de la Eutanasia del Partido Socialista Obrero Español

necesitan de algún tipo de ayuda y asistencia para desarrollar sus actividades diarias. La dependencia podría también estar originada o verse agravada por la ausencia de integración social, relaciones solidarias, entornos accesibles y recursos económicos adecuados para la vida de las personas mayores".

Por tanto, para delimitar, el concepto de discapacidad se puede decir que es un concepto multidimensional en la que concurren ciertos factores para ser definida se tiene que atender a los en términos físicos, mentales o emocionales, jurídicos, económicos y sociales.

Por otra parte, la discapacidad no se refiere sólo a criterios funcionales o clínicos, sino también incluyen criterios físicos, mentales, económicos en este último sentido manifiesta el autor Sempere (2006): "baste pensar que quienes más dependencia real tienen son las personas que suman a sus limitaciones psicofísicas un entorno económicamente débil” y, también socioculturales de la gente mayor con dependencia, enfermos o personas con discapacidad.

Por lo que, se entiende que es primordial que exista una innovación y la emergencia de nuevos sistemas que hayan tenido lugar en este campo de atención a la discapacidad y con la probabilidad que puedan concurrir hacia la dirección de soluciones privadas.

Por tanto, la problemática de toda esta evolución es como mantener el nivel de protección y cómo satisfacerlo porque existen en algunos casos vacíos en la demora de su aplicación, de las a Administraciones ya, que en este punto vista lo más importante es la protección social ${ }^{1}$ en este sentido cabe mencionar al autor Nores (2007) que manifiesta: "ofrece servicios continuados de carácter personal y sanitario desde un enfoque biopsicosocial", por lo que se entiende que los elementos a proteger de las personas discapacitadas son los cuidados prolongados así como los cuidados prolongados, el sistema sanitario, las pensiones.

Se articula como una prestación incluida en la cartera de servicios del Sistema Nacional de Salud, que se prestará por lo tanto en condiciones de igualdad para todos los ciudadanos, pero que, al contrario de lo que sucede con el tratamiento de la muerte digna, no se incluye en el código deontológico de las profesiones sanitarias y, en consecuencia, permite la objeción de conciencia. En este sentido cabe mencionar León (1997) que señala: "sería el análisis de las ideas que están en la base de las reclamaciones de autodeterminación de la propia muerte, la eutanasia por falta de "calidad de vida" o por 
la "inutilidad social" de la persona". Entiendo así que la persona se siente inútil ante la sociedad. O por no poder atender a las necesidades básicas de la vida diaria.

Todo ello con las suficientes garantías para garantizar que quien solicita la prestación de ayuda para morir es autónoma, libre de presiones, está suficientemente informada y que su decisión es consciente y mantenida en el tiempo. En este sentido, se exige mayoría de edad y plena capacidad de obrar para solicitar la ayuda para morir, que puede realizarse mediante documento de instrucciones previas o equivalente o no, y se contempla la existencia de una Comisión de Control y Evaluación que ha de controlar todo el proceso de forma previa y posterior a la muerte.

Al hilo, también cabe señalar que el legislador Socialista deja a los menores fuera del ámbito de la Proposición de Ley de la Eutanasia puesto que el tutor del menor o los padres no afrontan tal situación. Como señala la autora Castillo (2003): "La institución de la patria potestad emerge como una de las consecuencias de la filiación. En efecto el artículo 154 del Código civil en su párrafo primero, dispone que "los hijos no emancipados están bajo la potestad del padre y la madre”. En la misma actuación representativa, está el tutor, que se encuentra mencionado en el artículo 215 párrafo $1^{\circ} \mathrm{y}$

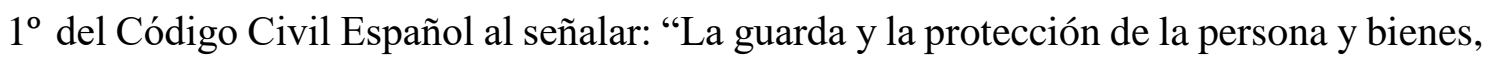
se realizará, en los casos que proceda, mediante: $1^{\circ}$ La tutela".

En coherencia con todo ello, esta Proposición de Ley Orgánica también contempla la necesaria despenalización de las conductas eutanásicas, modificando a tal efecto el art. 143.4 del Código Penal Español.

En este sentido se tendría que reformar el artículo 143.4 del Código Penal Español que señala: "El que causare o cooperare activamente con actos necesarios y directos a la muerte de otro, por la petición expresa, seria e inequívoca de éste, en el caso de que la víctima sufriera una enfermedad grave que conduciría necesariamente a su muerte, o que produjera graves padecimientos permanentes y difíciles de soportar, será castigado con la pena inferior en uno o dos grados a las señaladas en los números 2 y 3 de este artículo" y, siguiendo con el mismo artículo 143.1 y 2 señala: “2. Se impondrá la pena de prisión de dos a cinco años al que coopere con actos necesarios al suicidio de una persona, y 3. Será castigado con la pena de prisión de seis a diez años si la cooperación llegara hasta el punto de ejecutar la muerte".

Al hilo cabe mencionar la Sentencia de la Audiencia Provincial de Zaragoza de 19 de abril (Sección Sexta) (Sentencia 85/2016) (Ponente: Blasco Obede, Rubén) La Ley 36087/2016 Rec 40/ 15 en sus Fundamentos de Derecho Segundo señala: “ Los R. Fac. Dir. UFG, v. 42, n. 2, p.156-171, maio/ago. 2018 
La aplicación de la eutanasia en España: un estudio sobre la proposición de la Ley Ogánica de la Eutanasia del Partido Socialista Obrero Español

hechos son muy claros y no admiten duda alguna, pues desde un primer momento han sido reconocidos por el procesado, que nunca ha negado su participación activa en la muerte de su madre para auxiliar y cumplir su voluntad de suicidarse.

El deseo de suicidarse queda completamente probado, no solo por las manifestaciones del procesado, sino también por la nota manuscrita por aquella, nota cuya autoría se ha atribuido a la misma, como ratifica el dictamen pericial caligráfico practicado, estando también corroborado ese deseo por el propio mecanismo ideado por ella para quitarse la vida", Por lo tanto, se entiende que no será una muerte buena si la persona no accede ella misma a la eutanasia bajo su propia decisión y el legislador del Partido Socialista debe considerarlo como inducción al suicidio.

\section{LOS DISTINTOS TIPOS DE EUTANASIA}

En primer lugar, cabe menciona la eutanasia activa en la que, un sujeto, de una forma u otra, realiza conductas de matar al enfermo, no tratándose simplemente de dejarle morir. Esta forma de eutanasia contempla a su vez dos clasificaciones:

Por un lado, está la eutanasia activa indirecta que consiste en la causa de la muerte indolora a petición del afectado cuando éste es víctima de enfermedades incurables progresivas a través de medios directamente encaminados a provocar la muerte del enfermo.

Asimismo, cabe señalar, la eutanasia pasiva que consiste en la omisión de determinadas medidas que sólo sirven para prolongar artificial o innecesariamente la vida, cuya aplicación en contra de la voluntad del paciente podría incluso constituir un delito contra su libertad o su integridad moral, en el mismo sentido puede considerarse un abandono se trata de la falta de atención adecuada a las necesidades del enfermo y su familia. Entre sus motivos destacan la falsa idea de que "ya no hay nada que hacer" y una formación insuficiente en cuidados paliativos, así como el miedo o sensación de fracaso del profesional. Es el otro extremo de la obstinación y constituye, igualmente, una falta deontológica y una mala práctica médica. Probablemente muchas de las demandas de la eutanasia son realmente deseos anticipados de morir, que pueden tener su origen en un mayor o menor abandono por parte de quienes deberían atenderle profesional y humanamente. 
Y por último cabe mencionar, aunque hace años que se esta practicando en los hospitales de España la eutanasia pura que consiste que en la ayuda al bien morir mediante el uso de medios paliativos en el enfermo terminal.

\section{EL CONSENTIMIENTO INFORMADO A LA PERSONA QUE ESTÁ EN FASE TERMINAL O FAMILIARES}

La Ley 41/2002² de 14 de noviembre, Básica Reguladora de la Autonomía del Paciente y en Derechos y Obligaciones en materia de Documentación Clínica, contempla la información clínica del paciente. En este sentido, el derecho a la información está regulado en sus artículos $4^{3}$ y $5^{4}$, quien debe informar al paciente, a quién se le debe informar, de qué forma se debe informar y su contenido de información, junto con el derecho a no saber, y sus excepciones. De esta forma, su antecedente fue la Ley 14/1986, de 25 de abril, General de Sanidad, donde se expone en su artículo 10 "Todos tienen los siguientes derechos con respecto a las distintas administraciones públicas: 4. A ser advertido de si los procedimientos de pronóstico, diagnóstico y terapéuticos que se apliquen pueden ser utilizados en función de un proyecto docente o de investigación, que, en ningún caso, podrá comportar peligro adicional para su salud. En todo caso será imprescindible la previa autorización y por escrito del paciente y la aceptación por parte del médico y la Dirección del correspondiente Centro Sanitario", quedando esto reiterado en la Ley 21/2000, de 29 diciembre, de Cataluña, de Derechos de Información Concerniente a la Salud a la Autonomía del Paciente y a la Documentación Clínica, por lo que esta ley fue el inicio de las demás leyes autonómicas de España.

En este sentido, la autora Sánchez Gómez (2014) manifiesta: “El usuario de los servicios sanitarios, no solo demanda del facultativo unos cuidados adecuados y conformes según los medios y estado de la ciencia a fin de conseguir su curación, sino ser informado de manera completa y clara sobre los aspectos relativos a su estado de salud y la actuación que precisa", al hilo el Tribunal Supremo de España ${ }^{5}$ entiende: "puesto que la actuación decisoria pertenece al enfermo y afecta a su salud y como tal no es quien le informa si no él quien a través de la información que recibe, el que adopta la solución más favorable a su interés".

Por tanto, se entiende que el legislador socialista y los demás legisladores de los grupos políticos deberán modificar la proposición de ley sobre la Eutanasia, pues es importante el consentimiento del enfermo o, de sus familiares, a no ser que este en casa 
La aplicación de la eutanasia en España: un estudio sobre la proposición de la Ley Ogánica de la Eutanasia del Partido Socialista Obrero Español

y sea mediante un documento escrito pactado entre el enfermo terminal y su cuidador o familiar.

\section{LAS RAZONES QUE PROPUGNA EL PARTIDO SOCIALISTA OBRERO ESPAÑOL}

5.1. Porque es un hecho que existen personas que se enfrentan a situaciones de gran sufrimiento con un pronóstico irreversible de muerte.

5.2. Porque estas situaciones no se corrigen únicamente con el acceso universal a los cuidados paliativos, como se había mencionado anteriormente los cuidados paliativos pueden encarecer la prolongación de vida de la persona enferma en estado de fase terminal

5.3. Porque el Estado no puede permanecer al margen de esta realidad. En este sentido el legislador socialista atiende a que la figura jurídica de la eutanasia se legalice.

5. 4. Porque la respuesta del Estado debe hacerse con garantías y seguridad.

5. 5. Porque existe fundamento legal para dar este paso.

5.6. Porque se trata de ser sensible a la situación de sufrimiento insoportable que padecen algunas personas.

5.7. Porque para morir con dignidad es preciso contar con la ayuda de un profesional. Por lo que esta propuesta de Ley Orgánica se modificaría parcialmente

5.8 Porque existe una sensibilización en la sociedad española, al hilo cabe mencionar que también tienen decisión los distintos grupos del arco parlamentario tanto en el Congreso de Diputados como en el Senado Español.

5.9. Porque hemos adquirido el compromiso de regular la Eutanasia, por lo que el Partido Socialista Obrero Español no llevaba como elemento dentro de su programa político.

En Conclusión, la Propuesta de la Ley Orgánica es innovadora ante los nuevos retos de la sociedad española, simplemente como especifica su punto 7 “. Porque para morir con dignidad es preciso contar con la ayuda de un profesional",

\section{LA APLICACIÓN DE LOS CUIDAdOS PALIATIVOS COMO SUCEDÁNEO A LA EUTANASIA EN ESPAÑA}


Por tanto, observando el Ordenamiento Jurídico español se ha hecho la connotación de la existencia de sujetos en situación de discapacidad atendiéndolos así desde hace tiempo, cierto es que la protección social dispensada a estas personas que se encuentran en estas situaciones era una situación insuficiente y parcial, por la dispersión de normas con lo que conlleva la obligación de unir una normativa dispersa con un nutrido conjunto de normas jurídicas, emanadas por distintos poderes tanto estatales como autonómicos oscureciendo así la cobertura, de los cuidados paliativos solamente era el paciente quien toma la decisión, los familiares, tutores, o si están a cargo de alguna institución tutelar estatal o privada al hilo cabe mencionar al autor Seoane (2006) que señala: "El Convenio de derechos humanos y biomedicina introdujo a finales de la pasada década la regulación de las instrucciones previas o voluntades anticipadas (art. 9: deseos expresados anteriormente) en el sistema jurídico español. Su desarrollo legislativo comenzó a nivel autonómico (la pionera fue la Ley catalana 21/2000, de 29 de diciembre, sobre derechos de información concernientes a la salud, a la autonomía del paciente y a la documentación clínica) y ha seguido, a nivel estatal, con la Ley 41/2002, de 14 de noviembre, básica reguladora de autonomía del paciente y de los derechos y obligaciones en materia de información y documentación sanitaria, y a nivel autonómico, con abundantes leyes y decretos, tanto de índole general como específicamente destinados a regular diversos aspectos de las instrucciones previas o voluntades anticipadas".

Al hilo cabe mencionar la Sentencia del Juzgado de lo Contencioso Administrativo No7 Barcelona (Sentencia: 82/2016) (Ponente: Maestro Salcedo Andrés) La Ley 46769/2016. Rec.427/2014 en sus Fundamentos Jurídicos de Derecho Tercero señala: “A mayor abundamiento, el informe del ICAM (organismo imparcial) es concluyente en el sentido de entender que en ningún momento se le practicó al paciente una eutanasia activa, sino que, se le aplicó un tratamiento paliativo y que dado el estado avanzado de la enfermedad (cáncer) de aquél, no podía evitarse su muerte. Asimismo, concluye que los tratamientos administrados en todo momento al paciente fueron los adecuados a la situación clínica terminal que presentaba aquél, y que la atención prestada, en suma, se ajusta a la normo praxis asistencia o de eutanasia activa”.

De esta forma, la manera de actuar de los facultativos que asistieron al paciente de autos, fue ajustada a la "lex artis" y adecuada, atendiendo a las circunstancias del caso, sin que en ningún momento podamos hablar de desatención médica". De esta forma, se puede decir que se practica en España los cuidados paliativos, sedando a las personas en enfermedad terminal, como sucedáneo de la eutanasia, por lo que intenta el R. Fac. Dir. UFG, v. 42, n. 2, p.156-171, maio/ago. 2018 
La aplicación de la eutanasia en España: un estudio sobre la proposición de la Ley Ogánica de la Eutanasia del Partido Socialista Obrero Español

Partido Socialista Obrero Español legalizar estos últimos momento de la vida de la persona siempre bajo consentimiento de la misma o sus familiares o tutores la eutanasia.

\section{LOS ELEMENTOS JURÍDICOS PARA LA MODIFICACIÓN DE LA CONSTITUCIÓN ESPAÑOLA DE 1978}

La Constitución Española de 1978 es una de las pioneras cuando cita en su artículo 49: "Los poderes públicos realizaran una política de previsión, tratamiento, rehabilitación e integración de los disminuidos físicos, sensoriales y psíquicos, a los que prestarán la atención especializada que requieran y los ampararán especialmente para el disfrute de los derechos que este Título otorga a todos los ciudadanos", por lo que esto se traduce en una protección integral de las personas con discapacidad tanto de previsión, tratamiento, rehabilitación e integración atendiendo a la atención especializada que requieran que estos requieran.

Así, lo que cambio en estos últimos años ha sido la propia percepción de la sociedad acerca de cómo y quiénes han de proteger ese riesgo, actualizándose con gran intensidad en nuestra sociedad en este tiempo debido a la aparición de situaciones de discapacidad desde estos últimos años con el envejecimiento de la sociedad y enfermedades, en España.

Por lo que, es válida la eutanasia mediante la aplicación del artículo 10.1 de la Constitución Española que señala: "La dignidad de la persona, los derechos inviolables que le son inherentes" al hilo la autora Romero (2009) manifiesta: "Es evidente que, en el marco de la dignidad humana y del libre desarrollo de la personalidad, está inmerso el derecho de toda persona".

Por otro lado, el artículo 18.1 de la Constitución Española señala: "Se garantizará el derecho a la intimidad personal y familiar" al hilo la autora Romero (2009) manifiesta: "el derecho a la intimidad implica una facultad positiva o, en otras palabras, la de exigir a los poderes públicos que adopten las medidas necesarias para que cada persona pueda conocer efectivamente aspectos fundamentales de su propia vida".

Por tanto, se entiende que el fundamento jurídico del artículo 18 de la Constitución Española aborda analógicamente la facultad de decidir de la persona que esta en la fase terminal de su vida para decidir sobre la eutanasia, eso sí como menciona el Proyecto de la Ley Orgánica que sea mayor de edad "se exige mayoría de edad". 
Por otra parte, el artículo 15 de la Constitución Española señala: “Todos tienen derecho a la vida y a la integridad física y moral”, como se había mencionado anteriormente los cuidados paliativos se practican en las personas terminales en la actualidad contraviniendo este artículo, por lo que el legislador español una vez aprobada la eutanasia debería modificarlo. Y por último cabe mencionar el artículo 14 de la Constitución Española que señala: "Los españoles son iguales ante la ley, sin que pueda prevalecer discriminación alguna por razón de religión", por lo que el legislador socialista entiende que se puede aplicar la eutanasia a todas las religiones con el previo consentimiento de la persona enferma terminal o, con el consentimiento de sus familiares.

\section{CONCLUSIONES}

La eutanasia el legislador socialista dejo al margen a los menores de edad, aplicándose, los cuidados paliativos.

El artículo 143.4 del Código Penal Español se tiene que modificar, aunque como menciona la proposición de Ley Orgánica de la Eutanasia lo debe de realizar un profesional de la medicina.

El profesional de la medicina debe estar especializado en la aplicación de la Eutanasia.

La eutanasia debe ser aplicada según la Lex Artis de la medicina pues se tiene que recordar que el médico ésta formado para salvar vidas.

Los cuidados paliativos serían aplicable a menores lo que la Proposición de Ley no ésta en igualdad respecto a las personas mayores.

La eutanasia debe de atenerse a las convicciones religiosas, aunque la persona mayor de edad este bajo la tutela de las instituciones estatales o privadas siempre que tenga capacidad de obrar.

La eutanasia debe de ser aplicada si la persona enferma en fase terminal lo acepta mediante consentimiento informado o su familia.

\section{BIBLIOGRAFÍA}

CASTILLO MARTÍNEZ, Carolina (2003) (Dir.: Llopís Giner, Juan Manuel). La patria potestad, en AAVV, Curso Básico de Derecho de Familia y sucesiones. Editorial: Práctica de derecho. Sedaví-Valencia.

EAMON O’SHEA (2003). La mejora de la calidad de vida de las personas dependientes. Consejo de Europa. envejecimiento.csic.es/documentos/documentos/oshea-mejora-01.pdf. 
La aplicación de la eutanasia en España: un estudio sobre la proposición de la Ley Ogánica de la Eutanasia del Partido Socialista Obrero Español

LEÓN CORREA, Francisco Javier (1997). EI Debate sobre la Eutanasia y la medicina actual. Revista Ars Brevis No3 Editorial Anuari de la Càtedra Ramón Llull Blanquerna Barcelona.

NORES TORRES, Luis Enrique (2007). (Coord.: Roqueta Buj, Remedios). Servicios (III): El Servicio de Atención Residencial, en AA.VV., La protección de la dependencia. Comentarios a la Ley 39/2006, de 14 de diciembre, de Promoción de la Autonomía Personal y Atención a las personas en situación de dependencia. Ed.: Tirant lo Blanch. Valencia.

PINHEIRA PEREIRA, Américo José (2016). (Vol. $8 \mathrm{~N}^{\text {²}}$ ). Ensaio sobre a Eutanásia Editorial: Universidade Católica de Petrópolis, Petrópolis, Rio de Janeiro (Brasil).

RODRÍGUEZ CARRERO, Gregorio (2008). (Dir. Antonio Vicente Sempere Navarro) Bases demográficas de la dependencia. Población en situación de dependencia y cuidados informales en AA.VV., Comentario Sistemático a la Ley de Dependencia. Ley 39/2006, de 14 de diciembre, de Promoción de la Autonomía Personal en Situación de Dependencia y Normas Autonómicas. Editorial Aranzadi. S.A. Cizur Menor (Navarra).

ROMERO COLOMA, Aurelia María (2009). Identidad Genética frente la Intimidad. Diario La Ley, No 7199, Sección Tribuna, 18 de Junio de 2009, Año XXX, Ref. D-221, Editorial La Ley. Las Rozas (Madrid).

SÁNCHEZ GÓMEZ, Amelia (2014). La información al paciente y el consentimiento informado en el derecho español. Referencia legal y jurisprudencial. La praxis Médica. Revista Doctrinal Aranzadi Civil-Mercantil núm. 8/2014 parte Estudio. Cizur Menor. (Pamplona): Aranzadi.

SEOANE, José Antonio (2006). (Vol. 14. n²) Derecho y planificación anticipada de la atención panorama jurídico de las instrucciones previas en España Editorial Asociación Juristas de la Salud http://www.ajs.es/revista-derecho-y-salud.

Constitución Española y Constitución Europea. (2006). Consejo General de la abogacía Española. Editorial La Ley, Las Rozas (Madrid).

Código Civil (2006). Edición Especial. Consejo General de la abogacía Española. Editorial La Ley, Las Rozas (Madrid).

Código Penal. (2006). Edición Especial. Consejo General de la Abogacía Española. Editorial: La Ley, Las Rozas (Madrid).

Ley 14/1986, de 25 de abril, General de Sanidad; Rango Ley Ordinaria; Publicado Boletín Oficial del Estado (BOE) Madrid. N102 p. 15207. TOL.56.876 Tirant Online.

Ley 21/2000, de 29 diciembre, de Cataluña, de Derechos de Información Concerniente a la Salud a la Autonomía del Paciente y a la Documentación Clínica; Rango: Leyes y 
Decretos Autonómicos; Publicado Boletín Oficial del Estado (BOE) Madrid. N 29, p.41121. TOL.210.779. Tirant Online.

Ley 41/2002, 14 de noviembre, Básica Reguladora de la Autonomía del Paciente y en Derechos y Obligaciones en materia de Documentación Clínica; Rango Ley Ordinaria; Publicado Boletín Oficial del Estado (BOE) Madrid. N.274.p 4012. TOL. 215.524 Tirant Online.

Sentencia del Tribunal Supremo 11 de abril (Sala de lo Civil, Sección 1a) núm. 99/2013 (Ponente: Excmo. Sr. José Antonio Seija Quintana). RJ 2013/3384. Aranzadi.

Sentencia del Tribunal de Navarra 11 de enero de 2013 (Ponente: María Jesús Azcona Labiano) JUR 2014\44311. Aranzadi.

Sentencia del Juzgado de lo Contencioso - Administrativo $\mathbf{N}^{\mathbf{0} 7}$ Barcelona 29 de marzo de 2016 (Sentencia: 82/2016) (Ponente: Maestro Salcedo Andrés) La Ley 46769/2016. Rec.427/2014.

Sentencia de la Audiencia Provincial de Zaragoza de 19 de abril (Sección Sexta) (Sentencia: 85/2016) (Ponente: Blasco Obede, Rubén) La Ley 36087/2016. Rec 40/ 15.

Artigo recebido em 01 de outubro de 2018 e aceito em 06 de novembro 2018

${ }^{1}$ Constitución Española y Constitución Europea. (2006). Consejo General de la abogacía Española Editorial La Ley. Las Rozas (Madrid). pps 23-24 en su artículo 41 señala: "Los poderes públicos mantendrán un régimen público de Seguridad Social para todos los ciudadanos que garantice la asistencia y prestaciones sociales suficientes ante situaciones de necesidad".

${ }^{2}$ Ley 41/2002, 14 de noviembre, Reguladora de la Autonomía del Paciente y en Derechos y Obligaciones en materia de Documentación Clínica; Rango: Ley Ordinaria; Publicado Boletín Oficial del Estado (BOE) Madrid. N.274.p 4012. TOL. 215.524. Tirant Online.

${ }^{3}$ El artículo 4 señala: "1. Los pacientes tienen derecho a conocer, con motivo de cualquier actuación en el ámbito de su salud, toda la información disponible sobre la misma, salvando los supuestos exceptuados por la Ley. Además, toda persona tiene derecho a que se respete su voluntad de no ser informada. La información, que como regla general se proporcionará verbalmente dejando constancia en la historia clínica, comprende, como mínimo, la finalidad y la naturaleza de cada intervención, sus riesgos y sus consecuencias. 2. La información clínica forma parte de todas las actuaciones asistenciales, será verdadera, se comunicará al paciente de forma comprensible y adecuada a sus necesidades y le ayudará a tomar decisiones de acuerdo con su propia y libre voluntad. 3. El médico responsable del paciente le garantiza el cumplimiento de su información. Los profesionales que le atiendan durante el proceso concreto también serán responsables de informarle". 
La aplicación de la eutanasia en España: un estudio sobre la proposición de la Ley Ogánica de la Eutanasia del Partido Socialista Obrero Español

\footnotetext{
${ }^{4}$ El artículo 5 señala: "1. El titular del derecho a la información es el paciente. También serán informadas las personas vinculadas a él, por razones familiares o de hecho, en la medida que el paciente lo permita de manera expresa o tácita.2. El paciente será informado, incluso en caso de incapacidad, de modo adecuado a sus posibilidades de comprensión, cumpliendo con el deber de informar también a su representante legal.3. Cuando el paciente, según el criterio médico que le asiste, carezca de capacidad para entender la información a causa de su estado físico o psíquico, la información se pondrá en conocimiento de las personas vinculadas a él por razones familiares o de hecho. 4. El derecho a la información sanitaria de los pacientes puede limitarse por la existencia acreditada de un estado de necesidad terapéutica. Se entenderá por necesidad terapéutica la facultad del médico para actuar profesionalmente sin informar antes al paciente, cuando por razones objetivas el conocimiento de su propia situación pueda perjudicar su salud de manera grave. Llegado este caso, el médico dejará constancia razonada de las circunstancias en la historia clínica y comunicará su decisión a las personas vinculadas al paciente por razones familiares o de hecho".
}

${ }^{5}$ Sentencia del Tribunal Supremo 11 de abril (Sala de lo Civil, Sección 1ª) núm. 199/2013 (Ponente: Excmo. Sr. José Antonio Seija Quintana). RJ 2013/3384. Aranzadi. 